\title{
A Robotic System FOR Hydrogel 3D PRINTING ON COMPLEX SURFACES
}

\author{
Levin Aleksandr, Vorotnikov Andrei, Parfenov Vladislav, \\ Hesuani Usef, Mironov Vladimir \& Poduraev Yuri
}
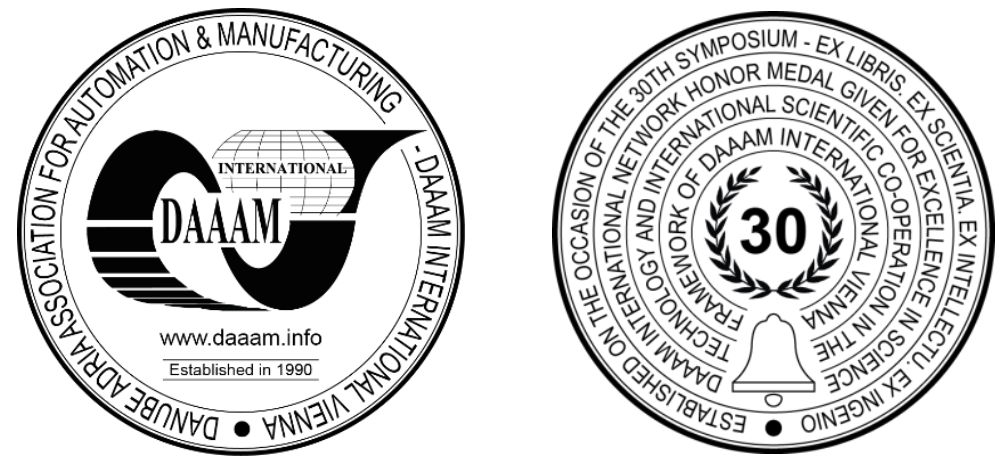

This Publication has to be referred as: Levin, A[leksandr]; Vorotnikov, A[ndrei]; Parfenov, V[ladislav]; Hesuani, U[sef]; Mironov, V[ladimir] \& Poduraev, Y[uri] (2020). A Robotic System for Hydrogel 3D Printing on Complex Surfaces, Proceedings of the 31st DAAAM International Symposium, pp.0793-0799, B. Katalinic (Ed.), Published by DAAAM International, ISBN 978-3-902734-29-7, ISSN 1726-9679, Vienna, Austria

DOI: $10.2507 / 31$ st.daaam.proceedings. 110

\begin{abstract}
The rapid development of sensor systems and the fall in the price of computing power in the near future will allow the creating adaptive robotic systems that can completely replace humans in operations that require high concentration, experience, and professionalism. The article presents the structure and software of a robotic system for in situ 3D bioprinting. The calculation of the material supply during bioprinting as a function of the linear velocity of the nozzle of the end effector is carried out. As well as its calculation depending on the surface area. This allows you to select the components of the developed robotic system. The development of an end effector for in situ 3D bioprinting is presented and the interaction of its components with each other is described. To ensure reliable operation of the system technical solutions are also demonstrated. An in vitro experiment was conducted on bioprinting cartilage tissue on a phantom of human femoral spongy tissue.
\end{abstract}

Keywords: bioprinting; automation; software development; robot control; extrusion.

\section{Introduction}

Today robotics is increasingly contributing to the development of many medical and biological disciplines[1]. Robots allow you to perform operations with speed and accuracy far exceeding the capabilities of humans [2]. Active research is underway to quantify these benefits [3],[4]. Methods are also being actively developed to further increase the accuracy of robots [5],[6]. We are working on creating user-friendly and intuitive interfaces for interaction between surgeons and robots [7]. One of the disciplines where robotics is actively implemented is bioprinting [8].

3D-bioprinting is a technology for layer-by-layer production of three-dimensional models on the cell surface, provided that the function and viability of cells are preserved. At the same time, bioprinting is always characterized by several important stages: creating a digital model of an organ or tissue, printing it in some way, and growing cells in an incubator [9],[10],[11]. Usually, after these stages, the organ is transplanted into the patient's body. When the material is applied directly to the patient's body to create or restore an organ, this is called in situ[8], or in vivo[12] bioprinting. 
Active research has been conducted in this direction for several decades [13]. One of the advantages of this approach over "classic" 3D bioprinting is that the patient's organ itself is an incubator for growing cells[14], and there is no need to perform a printed organ transplant operation. "Classic" 3D bioprinting refers to the printing of a structure from living cells using inkjet printing or in vitro lithography, followed by maturation in an incubator for some time and further transplantation to the patient.

Currently, in situ bioprinting is primarily used for the healing of organs and tissues such as cartilage, skin, and bones[15]. This is due to the relative ease of implementation. One of the important scientific problems in this field on the part of robotics is the choice of the method of applying the material to the body. There are several ways to solve it. For example, manual semi-automatic tools are used[16], or robotic systems[17],[18],[19]. In this work, the goal was to develop a robotic system that will allow bioprinting on the patient's body.

\section{The description of the developed robotic medical system structure for hydrogel bioprinting in situ}

When using robots for bioprinting in the structure of such systems, it is common that the end effector is a hydrogel extrusion tool. In this case, various methods of extrusion are used: using pneumatics or using an electric motor. Our system used an electric motor for hydrogel extrusion, as it is more convenient to use and does not require a compressor that takes up space. In bioprinting, the robot's movements and the volume of the material being squeezed out must be consistent. For this purpose, the following structure was identified and software was developed. The structure is shown in figure 1 .

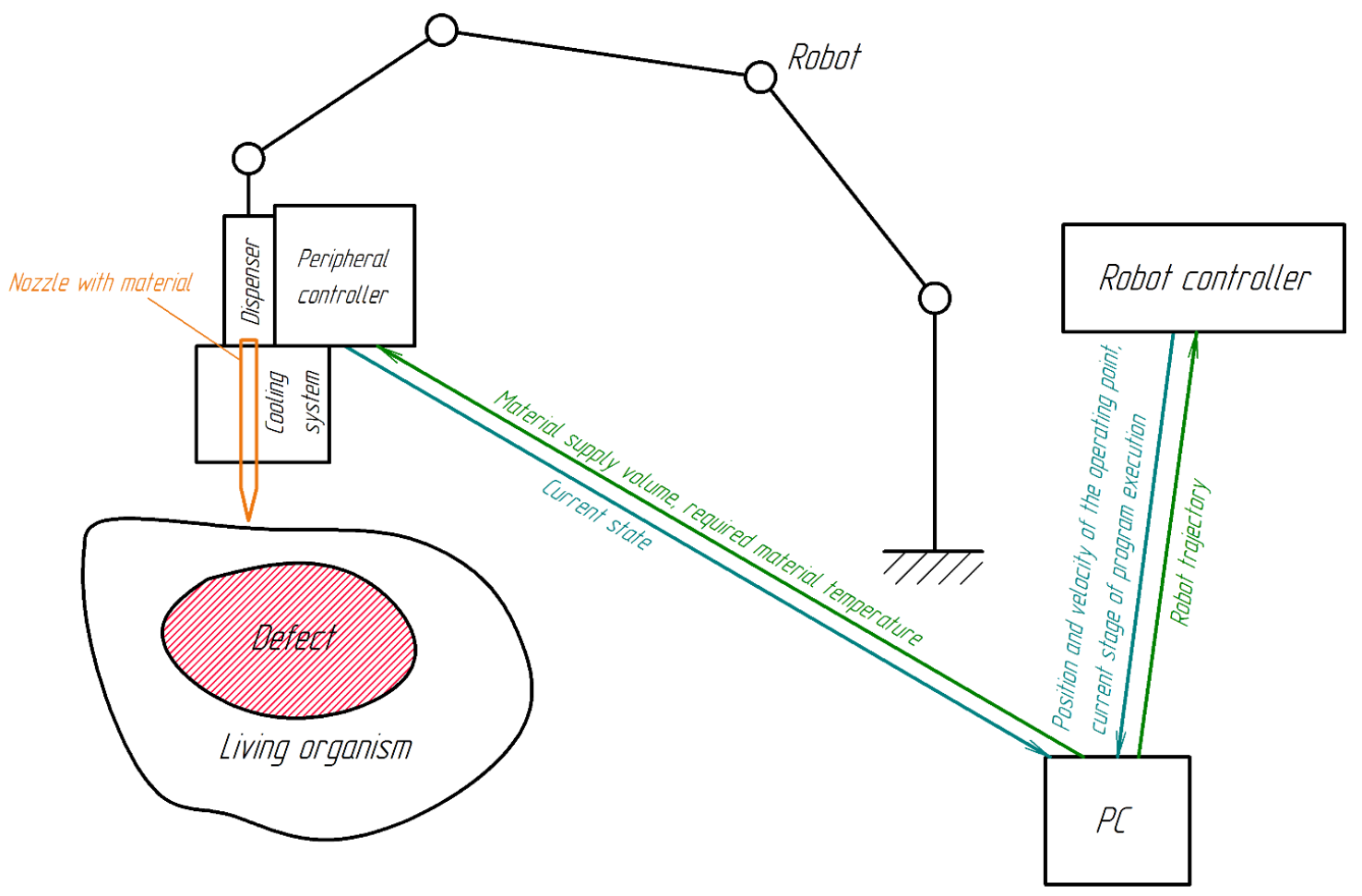

Fig. 1. The structure of the robotic medical system for hydrogel bioprinting in situ

This structure contains the following main components: robot, end effector, and control computer. The end effector performs the extrusion of the hydrogel, as well as maintains the desired temperature. The robot moves the end effector along a predetermined path. The control computer connects all components of the system to each other and it is also the interface between the operator and the system.

\section{Selection of the main components of the designed system}

The developing robotic medical system is designed to print material on uneven surfaces. At the same time, printing should be carried out quickly enough, since neutralized collagen will be used for printing, which cannot be stored for a long time. The print material defines some restrictions on the system being developed. For example, you can't print at a very high speed(more than $200 \mathrm{~mm} / \mathrm{s}$ ) because the material will change its behavior at this speed. Printing is supposed to be carried out by a mesh in the form of a circle. An approximate view of the mesh is shown in figure 2 a. When planning experiments on bioprinting, it is most preferable to set the following parameters. The lattice diameter $d$ mm. It is important that the edges of the circle may be curved. Mesh parameters: step $-s \mathrm{~mm}$, line thickness $-b$ mm, number of layers $-n$, layer height $-h$ microns. 
a)

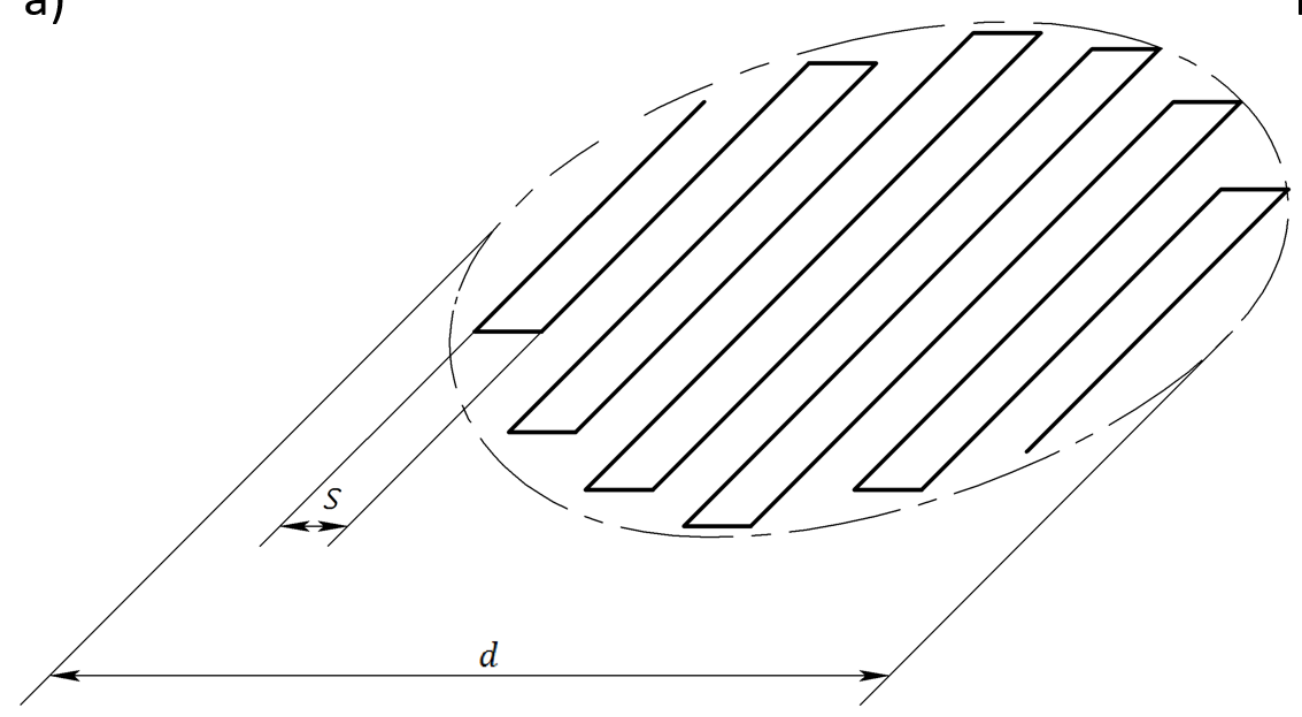

b)

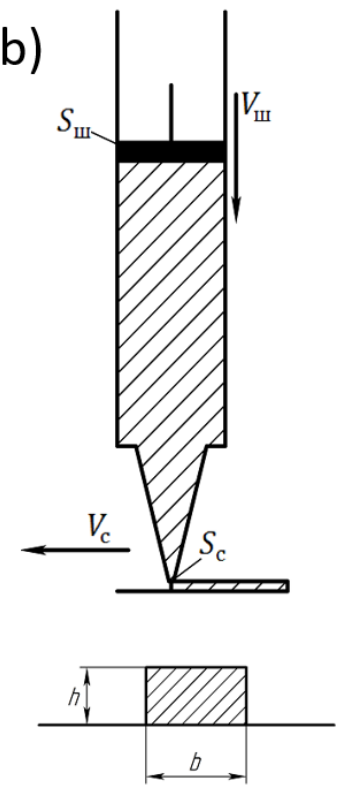

Fig. 2. Schematic representation of the path generation for the mesh, $a-$ the shape of the mesh, $b-$ the process of applying the material

Print parameters are calculated as follows. First, the linear velocity of the nozzle of the robot's end-effector $v$, which is necessary for such an operation, is determined. It can be found using the following formula:

$$
v=\frac{L}{t}
$$

where $\mathrm{L}$ is the path length. The figure shows that the path length can be found as follows:

$$
L=\left(\frac{d}{h}+1\right) * d * n
$$

where $d$ is the length of the shape side, $h$ is the print step, and $n$ is the number of layers. From this formula, it follows that for $\gg h$, the first term of the expression will be much larger than the second. The area of the shape is equal to:

$$
S=d^{2}
$$

It follows that the path length at $d \gg h$ is directly proportional to the print surface area. This rule is valid if the areas of the layers are the same. Thus, for a circular defect, the trajectory length will be equal to:

$$
L=\frac{\pi}{4}\left(\frac{d}{h}+1\right) * d * n
$$

where $\frac{\pi}{4}$ is the ratio of square and circle areas. Thus, the robot's speed range was obtained from these ratios: $v=$ $5 \ldots 60 \mathrm{~mm} / \mathrm{sec}$. When designing this system, it is assumed that the robot fully covers the required range of the required speed range, and also has a control system that can be connected directly to the PC. For such requirements, it is advisable to choose the KUKA KR iiwa 14 R820 as the robot. To be able to fully programmatically control the process of applying the material, we choose a dispenser with a rod that moves using of a screw transmission. At the same time, the maximum speed of the rod was first estimated. This speed is expressed from the following condition:

$$
V_{\mathrm{\amalg}} * S_{\mathrm{\amalg}}=V_{\mathrm{c}} * S_{\mathrm{c}}
$$

where $V_{\amalg}, S_{\mathrm{\Psi}}$ are the linear velocity and cross-sectional area of the syringe piston, respectively, and $V_{\mathrm{c}}, S_{\mathrm{c}}$ are the linear velocity and cross-sectional area of the nozzle, respectively. The parameters of the material application process are shown in figure 2 b. A $9 \mathrm{~mm}$ diameter syringe was used. Thus, the cross-sectional area of the material flow squeezed out of the nozzle is calculated as follows:

$$
S_{\mathrm{c}}=h_{\mathrm{c}} * w_{\mathrm{c}}
$$


where $h_{\mathrm{c}}$ is the layer height and $w_{\mathrm{c}}$ is the line thickness. Then the speed of the rod is found from the following expression:

$$
V_{\mathrm{\Psi}}=0,05 \ldots 1 \mathrm{MM} / \mathrm{c} \text {; }
$$

Most of the existing dispensers satisfy us in terms of linear velocity. The Fishman SDAV was chosen because this dispenser combines the precision of material extrusion and ease of operation. The developed robotic medical system for bioprinting includes a collaborative robot KUKA BR iiva 14 R820, a printing device, and a control computer. The main components of the printer are Fishman nozzle, cooling system, and control unit. A detailed description of the cooling system of the robot's end effector-a medical system for bioprinting is shown in figure 3 .

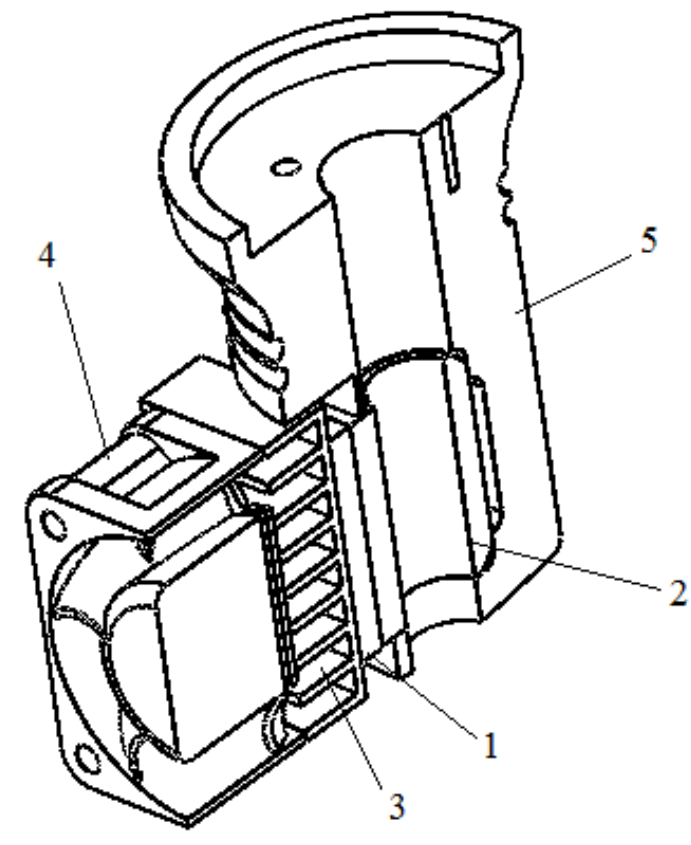

Fig. 3. The design of the cooling system

The cooling system consists of a metal part (2), an external housing (5), a Peltier module (1), a radiator (3), and a cooler (4). When the Peltier module is turned on, the metal part cools and the radiator heats up. For better heat exchange, the metal part and radiator are made of aluminum. Thus, the metal part cools the syringe, and the radiator gives off heat to the external environment. A fan is installed to speed up the heat output. The nozzle consists of a stepper motor, a rod, and a special attachment for the syringe piston. When voltage is applied to the motor windings, the piston moves, and if the syringe is filled without air voids, the material will either be squeezed out or raised into the syringe, respectively.

\section{The description of bioprinting software}

The printer controller operates according to the following algorithm. The program is in an infinite loop and is waiting for a message from the control computer, which contains information about how fast and how many steps the nozzle piston should move, and whether the cooling system should be turned on now or not.

In turn, the robot controller first sends the coordinates of the points that characterize the wound surface. Then an array of trajectory data is received from the control computer, which also includes information about the volume of the extruded material. The trajectory is a set of short lines. Then the program waits for the operator's permission to start printing. If the resolution is obtained, the robot starts moving along each line with a minimum smoothing radius, while maintaining a constant linear speed. This ensures that the material is applied evenly. At the same time, to ensure non-stop operation, commands are executed on the robot in asynchronous mode, that is, the internal robot controller responsible for interpolating the robot's movements is several commands ahead of the upper-level robot controller.

To synchronize the robot's movements and the extruded material from the nozzle, the following algorithm is used. During the execution of the trajectory, the robot sends a command to build the next line only after it passes the line point of the 2 commands back. To do this, the robot checks its current coordinate and compares it with the coordinate of the end of the current line. The program on the control computer is a graphical interface with the ability to start and stop the bioprinting process. It also provides the ability to manually control the printer. First, a trajectory for the plane is generated using SprutCam. In this way, we set the main printing parameters: the thickness of the filament, the printing speed, and the filling method. The bioprinting algorithm is as follows. 
When the user clicks the print start command, the program sends a message to the robot controller about the start of printing, and the robot controller sends back the points that characterize the print surface. The program decrypts the file with the trajectory and saves it as an array, while all lines are divided into short lines with a length of $1 \mathrm{~mm}$. Then, according to the loaded points, the trajectory is cut along the border. Then the surface equations are calculated from the points, and the trajectory is projected onto the resulting surface. This results in an array of lines that are already passed to the robot controller. After that, the program starts working as a link between the robot controller and the printer controller. The program sends messages about the delivery of material from the robot controller to the printer controller.

\section{In vitro experiment}

For the in vitro experiment, the previously described system was used, the appearance of which is shown in figure 4.

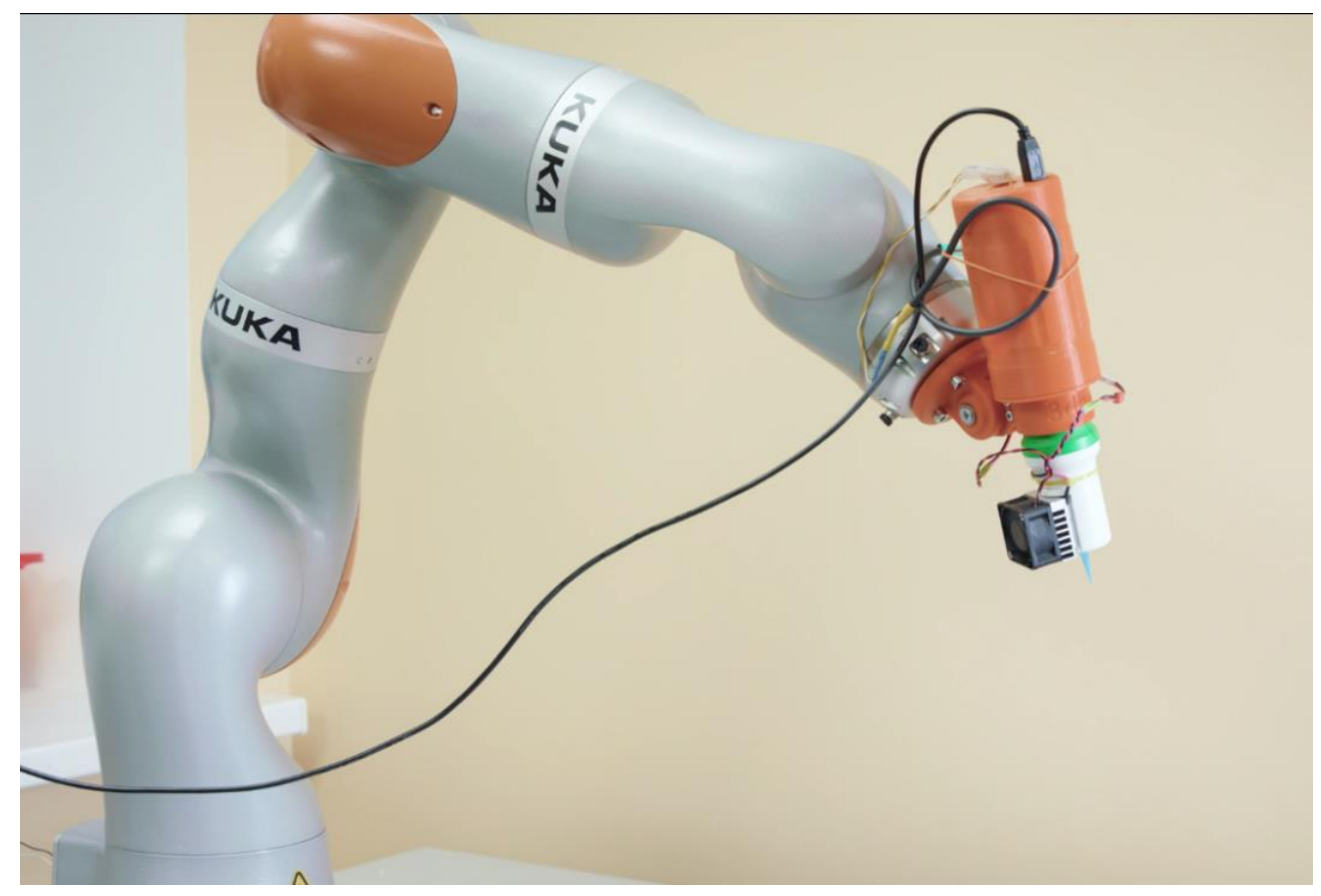

Fig. 4. The experimental setup of a robotic medical system for 3D hydrogel bioprinting in situ

An in vitro experiment was conducted to confirm the functionality of the developed system. To do this, a phantom of the spongy tissue of the human femur was made and a defect was made on it (figure 5). FlexFoam-iT 17 was chosen as the material for the sponge fabric phantom. Printing was carried out at a speed of $20 \mathrm{~mm} / \mathrm{s}$, a defect diameter of $15 \mathrm{~m}$, a lattice pitch of $1.5 \mathrm{~mm}$, a filament thickness of 800 microns, and a layer height of 400 microns.

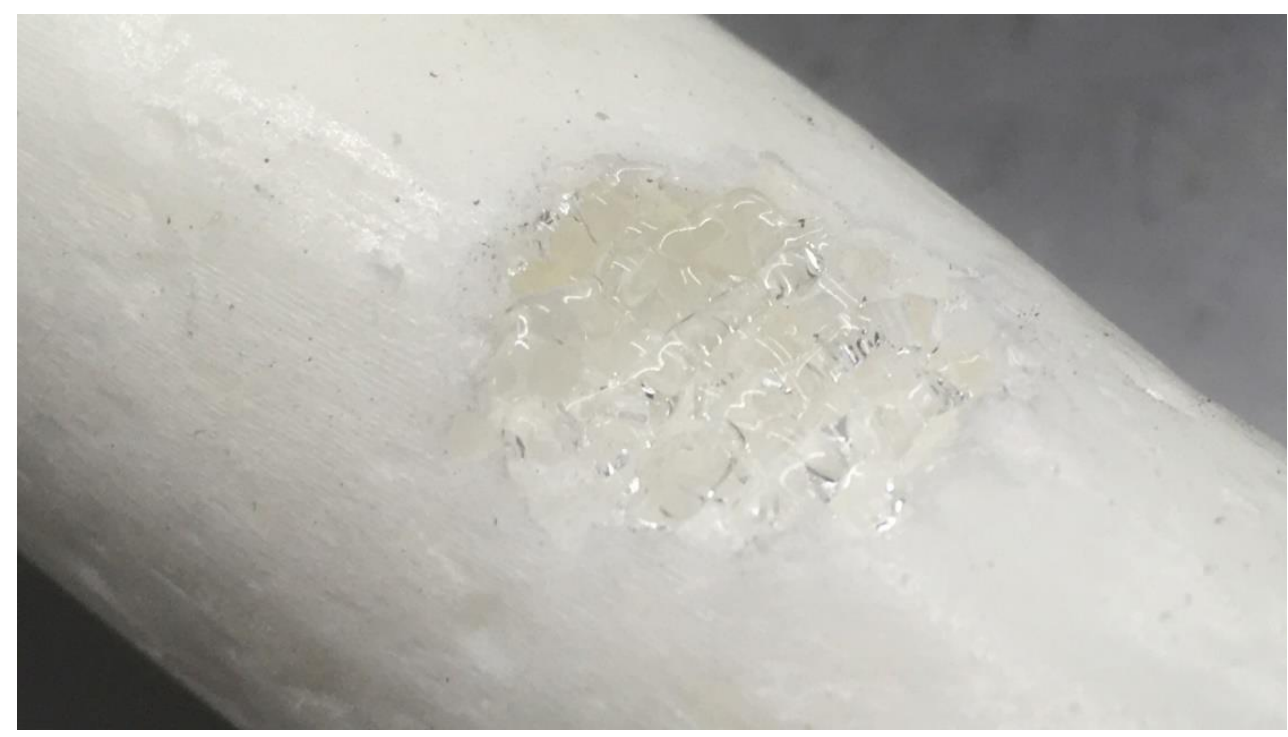

Fig. 5. The result of bioprinting on the femoral phantom 
The figure shows that the hydrogel completely filled the defect model with sufficient accuracy. The experiment confirmed the efficiency of the developed system. The next stage will be testing on laboratory animals.

\section{Conclusion}

As a result of this work, the main components of the robotic medical system were selected. They were determined based on the required printing parameters, as well as a calculation based on the expected operating mode of the system. Based on this component base, the design of the end effector was developed as the main component of this system. As a result, the scheme of work of the medical system was built and based on it, the software was created that allows you to flexibly manage the created system. One of the main problems was the coordinated operation of all electronic components of the system. For this purpose, software was developed for each electronic component of the system. The solution was a system that allows you to print on curved surfaces of the structure in the form of a circular grid.

In the future, it is planned to improve the hardware and develop an algorithm that allows you to automatically calculate the robot's trajectory for more complex surfaces.

\section{Acknowledgements}

Scientific Research by Educational Organizations in 2020-2022 Project under Grant NoFSFS-2020-0031

\section{References}

[1] Moldovan, N.I., Hibino, N., and Nakayama, K. (2017) "Principles of the Kenzan Method for Robotic Cell SpheroidBased Three-Dimensional Bioprinting," Tissue Engineering Part B: Reviews,Vol. 23, No. 3, pp. 237-244., ISSN 1937-3368, DOI 10.1089/ten.teb.2016.0322

[2] Solovyev, M., Vorotnikov, A., Klimov, D., Kovalskii, V., and Poduraev, Y. V. (2017) "Control System of the Articulated Arm Braking Mechatronic Machine (AABMM), pp. 1002-1009, DOI 10.2507/28th.daaam.proceedings.139

[3] Vorotnikov, A.A., Klimov, D.D.K., Melnichenko, E.A., Poduraev, Y. V., and Bazykyan, E.A. (2018) "Criteria for Comparison of Robot Movement Trajectories and Manual Movements of a Doctor for Performing Maxillofacial Surgeries," International Journal of Mechanical Engineering and Robotics Research, Vol. 7, No. 4, pp. 361-366., ISSN 22780149, DOI 10.18178/ijmerr.7.4.361-366

[4] Vorotnikov, A.A., Buinov, M.A., Bushuev, S. V., Poduraev, Y. V., and Chunihin, A.A. (2016) "Standard Deviation from the Average Cutting Velocity as a Criterion for Comparing Robot Trajectories and Manual Movements of a Doctor for Performing Surgical Operations in Maxillofacial Surgery," International Journal of Mechanical Engineering and Robotics Research, Vol. 7, No. 2, pp. 319-323., ISSN 22780149, DOI 10.18178/ijmerr.7.3.319323

[5] Vorotnikov, A., Romash, E., Isaev, A., Bashevskaya, O., Bianchi, G., and Poduraev, Y. (2016) "Uncertainty Estimation of Axes Direction Determination of Industrial Robot Using an Ellipsoid Concentration Model. in: pp. 0480-0486, DOI 10.2507/27th.daaam.proceedings.072

[6] Vorotnikov, A., Bashevskaya, O., Ilyukhin, Y., Romash, E., Isaev, A. V., and Poduraev, Y. (2016) Geometrical Approach for Industrial Robot Axis Calibration Using Laser Tracker. pp. 0897-0904., DOI 10.2507/26th.daaam.proceedings. 125

[7] Prokhorenko, L., Klimov, D., Mishchenkov, D., and Poduraev, Y. (2020) "Surgeon-robot interface development framework," Computers in Biology and Medicine. Vol. 120, pp. 103717., ISSN 00104825, DOI 10.1016/j.compbiomed.2020.103717

[8] Singh, S., Choudhury, D., Yu, F., Mironov, V., and Naing, M.W. (2020)" In situ bioprinting - Bioprinting from benchside to bedside?," Acta Biomaterialia, Vol. 101, pp. 14-25., ISSN 17427061, DOI 10.1016/j.actbio.2019.08.045

[9] Duan, B., Hockaday, L.A., Kang, K.H., and Butcher, J.T. (2013) "3D Bioprinting of heterogeneous aortic valve conduits with alginate/gelatin hydrogels," Journal of Biomedical Materials Research Part A, Vol. 101A, No. 5, pp. 1255-1264., ISSN 15493296, DOI 10.1002/jbm.a.34420

[10] Hockaday, L.A., Kang, K.H., Colangelo, N.W., Cheung, P.Y.C., Duan, B., Malone, E., et al. (2012) "Rapid 3D printing of anatomically accurate and mechanically heterogeneous aortic valve hydrogel scaffolds," Biofabrication, Vol. 4, No. 3, pp. 035005, ISSN 1758-5082, DOI 10.1088/1758-5082/4/3/035005

[11] Shafiee, A. and Atala, A. (2016)"Printing Technologies for Medical Applications," Trends in Molecular Medicine, Vol. 22, No. 3, pp. 254-265, ISSN 1471-4914, DOI 10.1016/J.MOLMED.2016.01.003

[12] Keriquel, V., Guillemot, F., Arnault, I., Guillotin, B., Miraux, S., Amédée, J., et al. (2010) "In vivo bioprinting for computer- and robotic-assisted medical intervention: preliminary study in mice," Biofabrication, Vol. 2, No. 1, pp. 014101, ISSN 1758-5082, DOI 10.1088/1758-5082/2/1/014101

[13] Sigaux, N., Pourchet, L., Breton, P., Brosset, S., Louvrier, A., and Marquette, C. (2019) "3D Bioprinting:principles, fantasies and prospects," Journal of Stomatology, Oral and Maxillofacial Surgery, Vol. 120, No. 2, pp. 128-132, ISSN 24687855, DOI 10.1016/j.jormas.2018.12.014 
[14] Ng, W.L., Chua, C.K., and Shen, Y.-F. (2019) "Print Me An Organ! Why We Are Not There Yet," Progress in Polymer Science, Vol. 97, pp. 101145, ISSN 00796700, DOI 10.1016/j.progpolymsci.2019.101145

[15] Malyshev, I.Y., Runova, G.S., Poduraev, Y. V., and Mironov, V.A. (2018) "Natural amelogenesis and rationale for enamel regeneration by means of robotic bioprinting of tissues in situ," Stomatologiya, Vol. 97, No. 2, pp. 58, ISSN 0039-1735, DOI 10.17116/stomat201897258-64

[16] Wu, Y., Ravnic, D.J., and Ozbolat, I.T. (2020) "Intraoperative Bioprinting: Repairing Tissues and Organs in a Surgical Setting," Trends in Biotechnology, Vol. 38, No. 6, pp. 594-605, ISSN 01677799, DOI 10.1016/j.tibtech.2020.01.004

[17] Ashammakhi, N., Ahadian, S., Pountos, I., Hu, S.-K., Tellisi, N., Bandaru, P., et al. (2019) "In situ three-dimensional printing for reparative and regenerative therapy," Biomedical Microdevices, Vol. 21, No. 2, pp. 42, ISSN 13872176, DOI 10.1007/s10544-019-0372-2

[18] Lipskas, J., Deep, K., and Yao, W. (2019) "Robotic-Assisted 3D Bio-printing for Repairing Bone and Cartilage Defects through a Minimally Invasive Approach," Scientific Reports, Vol. 9, No. 1, pp. 3746, ISSN 2045-2322, DOI 10.1038/s41598-019-38972-2

[19] Kahin, K., Khan, Z., Albagami, M., Usman, S., Bahnshal, S., Alwazani, H., et al. (2019) "Development of a robotic 3D bioprinting and microfluidic pumping system for tissue and organ engineering. B.L. Gray, H. Becker (Eds.), Microfluid. BioMEMS, Med. Microsystems XVII, SPIE, p. 25, ISSN 9781510623927, DOI 10.1117/12.2507237 